\title{
Lean Principles Implementation in Construction Management: A One Team Approach
}

\author{
Mahmoud Fahmy \\ mfahmy@qetaifanprojects.com \\ Qetaifan Project, Doha, Qatar
}

\begin{abstract}
Lean in construction is a relatively new approach in delivering construction projects. The Lean approach has been introduced to tackle the phenomenal construction projects delays and budget overruns. This paper shall be discussing how the implementation of the Lean approach in construction management processes can improve construction projects' delivery. The paper argues that the Lean approach in the construction industry context revolves around people rather than processes as in the manufacturing industry context. Therefore, the cornerstone of the Lean approach in construction is creating a One Team out of the multiple typical rivals in the construction industry: Owner, architect / engineer, operator and contractor with all taking into consideration the end user. While the 'Lean' concept is manufacturing tackled processes to achieve the 'Lean' goal; in construction the 'Lean' approach is tackling the interaction between people and entities. The interaction between people is addressed by the 'Lean construction' planning tool: The last planner system; while the interaction between entities is addressed by contractual forms of agreement with the most influential being: Alliance contracting. The paper uses a major infrastructure project 'Sydney Desalination Plant' which started in June 2007 and completed in January 2010 as a successful example of Lean approach implementation, although the term 'Lean' was never used then by the parties involved in the project. The paper draws on the first-hand experience of the author in the construction industry including the participation in the delivery of the 'Sydney Desalination Plant' used as example of a Lean project.
\end{abstract}

Keywords: Lean construction; Alliance contracting; Team collaboration

\section{INTRODUCTION}

Lean construction is a concept and approach that helps in delivering construction projects. It evolved due to the dire situation of the construction industry where the norm is: projects are delayed, and projects' budgets are overrun. However, it is worth noting that the term 'lean construction' is not limited to the construction phase of a project as the term might be perceived. 'Lean construction' is an overarching concept which encompasses both the design and construction phases of a project. There are number of definitions for 'lean construction' and this paper adopts the definition: "it is a way to design production systems to minimize waste of materials, time, and effort in order to generate the maximum possible amount of value" (Koskela et al., 2002). Meanwhile, it is important to highlight that the 'lean' concept evolved originally from the manufacturing industry to improve the manufacturing processes. While the goal in applying the 'lean' 
concept in both industries is one, eliminating waste and maximizing value, the nature of the two industries is different. This paper discusses a major unique characteristic in construction projects setting it apart of the manufacturing industry; which is the human component. In construction projects the direct interface of people of different levels in: intellect, risk appetite, organizational skills and process-driven approach is un avoidable, where in manufacturing to a far extent, automation played a major role in breaking the links of interface between individuals who have high level difference in professional/industry culture. Several 'lean construction' literature and textbooks touched on the fundamental impact of people factor in applying the 'lean' principles. One of many examples is Gary Santorella's book on Lean Culture where he mentions that the main reason for writing the book was "to draw a connection between how construction professionals act as leaders (both positively and negatively) and the subsequent affect their attitude and behavior has on productivity and waste" (Santorella, 2011). Additionally, in the construction industry the interface between multiple entities when each has its own motivation and goals is far complex than in the manufacturing industry where the end product is well defined, and uncertainties are almost eliminated. Therefore, while the 'lean' concept is manufacturing tackled processes to achieve the 'lean' goal; in construction the 'lean' approach is tackling the interaction between people and entities. The interaction between people is addressed by the 'lean construction' planning tool: The last planner system; while the interaction between entities is addressed by contractual forms of agreement with the most influential being: Alliance contracting. Both the last planner system and alliance contracting are founded on 'collaboration', which this paper refers to as 'A One Team Approach'.

\section{COLLABORATION}

If we to dissect the 'lean' principles in the construction industry, we will recognize that it is founded on the term 'collaboration'. The reason why collaboration falls at the core of 'lean' principles is the nature of the industry, being a people driven industry. However, people are present in any project in two dimensions:

- Individual dimension: Each present by his own set of skills, personality and objectives.

- Entity dimension: Each group of individuals representing the entity, which they belong to.

The 'lean' concept in simple terms is about finding the way (set of processes) which allows project teams to collaborate efficiently in order to eliminate waste and maximize value. The complexity in this simple statement lies in the word 'project teams' i.e. people with their two dimensions present at the project. The first dimension (individuals) is dealt with (firstly) through people skills / soft skills, which cannot be structured in a process to manage the individuals' outcome. Therefore, the 'lean' in construction introduced the 'pull' planning concept opposing the conventional 'push' method as a process to address collaboration at the individual level. The 'pull' planning concept implemented by the 'last planner system' is out of the scope of this paper discussion. However, it is worth noting that 'collaboration', which is the core 'lean principle' is standing on two pillars (as illustrated in figure 1): The collaboration at the individuals' level and the collaboration at the entities level. 


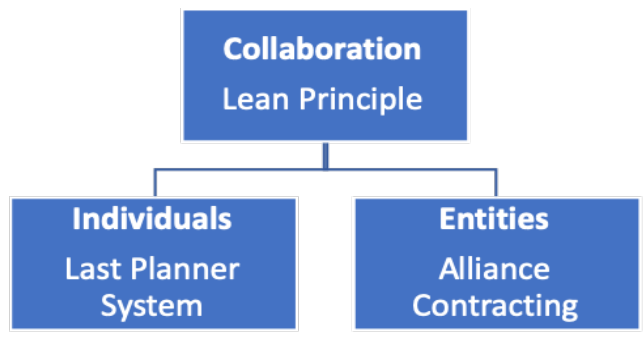

Figure 1: Collaboration pillars

\section{TRADITIONAL CONTRACTING}

A typical multidisciplinary / mega project involves the following entities:

- Owner

- Architect / Engineer

- Contractor

- Operator / Facility Manager

The traditional forms of contract in the construction industry have been:

- $\quad$ Design - Bid - Build

- Design \& Build

- Engineering Procurement Construction (EPC)

All three forms inherently and traditionally dealt with the organizations participating in a project as rivals. The reason being that when a project goes off-track, which is more often than not, each entity strives to prove that the other entity is solely responsible for the unfavorable outcome. This phenomenon is quite understandable for the following reasons:

- Individuals of each entity strive to protect their own entity interest.

- Individuals, regardless of which entity they belong to, strive to depart themselves from the project failures.

- The incidents contributing to the failure of the project are, most often, complex and have the capacity to be interpreted by each entity differently.

Accordingly, the traditional forms of contract launch projects are carried by rival entities rather than collaborating entities.

\section{ALLIANCE CONTRACTING}

'Alliance contracting', "also known as 'collaborative contracting' is a delivery framework for large multidisciplinary projects, focusing on a co-operative process which aims to promote openness, trust, risk and responsibility sharing, innovation, high performance and the alignment of commercial interests between parties who aim to deliver a project in a collaborative and constructive way," (Walsh, 2015). This offers a different approach, 'the one team approach' by bringing together the entities involved in a project under one umbrella. Accordingly, the 'alliance contracting' addresses the entities dimension by bringing them in a single domain within a framework allowing them to share benefits and risks. Alliance contracting has been implemented in the UK, 
Europe, Asia Pacific, Australia \& New Zealand with remarkable gains being achieved. Furthermore, there are discussions "that although the volume of alliance activity is growing, the potential benefits of such organizational arrangements are not being realized because participating firms are emphasizing short-term profit sharing and accountancy procedures to the detriment of the more long-term and, ultimately," (Ingirige \& Sexton, 2006). This paper is only discussing the first fold of 'alliance contracting', the shortterm fold, which in itself serves the 'lean' principles implementation as discussed in the different approach it has towards project problems.

\section{APPROACHING PROBLEMS}

Clearly, the traditional forms of contract and the 'alliance' form of contract shall result in a completely different approach in dealing with problems facing a project. In traditional contracting, each and every problem facing the project is approached as a dispute between the rival entities. Managing a dispute shifts the focus from resolving the problem to arguing which entity is at fault. While in 'alliance contracting', entities become one team focusing on problems at hand as a challenge facing the project. Table 1: Different approaches in addressing problems provides a comparison between the traditional and the alliance contracting:

Table 1: Different approaches in addressing problems

\begin{tabular}{|l|l|l|}
\hline & Traditional Contracting & Alliance Contracting \\
\hline Project Entities & Rivals & One Team \\
\hline Problem facing the project & Result in dispute & Treated as a challenge \\
\hline Goal & Identifying who is at fault & Eliminating the problem \\
\hline Outcome & $\begin{array}{l}\text { Unresolved problem leading to } \\
\text { further problems and waste }\end{array}$ & $\begin{array}{l}\text { Moving forward and sharing } \\
\text { gain / loss }\end{array}$ \\
\hline
\end{tabular}

\section{SUCCESSFUL EXAMPLE: SYDNEY DESALINATION PLANT}

Since late 1990's 'alliance contracting' has been used in mega construction projects in Australia and New Zealand. A successful example of which is the Sydney Desalination Plant started in June 2007 and completed in January 2010. The project owner, Sydney Water (a government owned entity at the time) made an alliance called 'White Water' with the contractor John Holland and the operator Veolia Water and launched the 1 billion AUD project to build 250 mega litter/day plant (expandable to 500 mega litter/day), which was a fast track project to tackle the critical low dam levels supplying Sydney with potable water. The alliance then contracted SKM (currently Jacobs) and Maunsell (currently AECOM) JV to undertake the engineering of the project. The project, which successfully provided over $15 \%$ of Sydney's potable water supply, was completed on time and under budget by 60 million AUD approximately. Although the term 'lean construction' was never used and the project planning system was the conventional CM method, yet the team collaboration and the one team approach were the main contributors to the project success. That was essentially achieved by introducing the benefit / risksharing concept with all entities including the design JV that was not part of the alliance. All entities agreed to bring their teams together, literally all wearing the same alliance shirt, 'White Water'. Each member of the team working on the project belonged to the 
project rather than to the entity, he/ she originally came from. Hence, the project was a success.

\section{CONCLUSION}

Collaboration is an obvious key to projects success. However, implementing collaboration and 'A One Team Approach' cannot be achieved without a proper contract framework, which facilitates the benefit / risk sharing between the entities involved in the project. The most reliable form of contract addressing collaboration, which is backed by record of accomplishment of successful projects, is the 'alliance contracting'. However, the challenge facing the implementation of this form of contract, in many parts of the world, is that the legal frameworks in public sector do not have this form of contracts.

\section{REFERENCES}

Ingirige, B. \& Sexton, M. (2006). Alliances in construction investigating initiatives and barriers for long-term collaboration. Research Institute of the Built and Human Environment, University of Salford, Salford, the UK.

Koskela, L., Howell, G., Ballard, G. \& Tommelein, I. (2002). The Foundations of Lean Construction. Research Gate.

Santorella, G. (2011). Lean Culture for the Construction Industry - Building Responsible and Committed Project Teams. P. xviii, CRC Press.

Walsh, P. (2015). Alliance Contracting: The Way Forward, Thomson Reuters. 